\title{
Evaluating the UK takeaway food environment around secondary schools: comparison of different methods
}

\author{
Ayyoub K. Taher ${ }^{1,2}$, Charlotte E L. Evans ${ }^{1}$ and Graham P Clarke ${ }^{3}$ \\ ${ }^{1}$ School of Food Science and Nutrition, Nutrition and Epidemiology Group, University of Leeds, Leeds, United \\ Kingdom, \\ ${ }^{2}$ Food and Nutrition Program, Environment \& Life Sciences Research Centre, Kuwait Institute for Scientific Research, \\ Safat, Kuwait and \\ ${ }^{3}$ School of Geography, University of Leeds, Leeds, United Kingdom
}

\begin{abstract}
Takeaway shops are more clustered around secondary than primary schools and UK planning policies to limit takeaways show poor implementation against international examples and good practice statements. A major concern is that, worldwide, there are no standardised measures used to measure the food environment around homes, schools, work or any other facilities. This study aims to examine the differences in using different methods to evaluate the food environment particularly around secondary schools in the Avon region in the UK. Geographical Information System (GIS) was used to locate all schools and takeaways in the region and to measure the density and proximity scores, applying both road network and straight-line methods. In addition, the Hansen Index was used to measure the accessibility score of each schools to all takeaways in the region (not just the nearest). All of the nonparametric statistical analysis tests including Wilcoxon test, agreement (Kappa statistic) test and correlation test were carried out using Stata software version 15.0. It was observed that more than $50 \%$ of the schools had no takeaway shops within 200,400 , and 600 metres when the road network buffer was used. Statistical differences were observed between the road network and the straight-line methods. For example, the median of the difference between the straight-line and road network density within 1000 metres was 4.1 (CI 2.6, 5.9; $\mathrm{P}<0.001$ ). The median of the difference between the road network and straight-line proximity was 203.2 (CI 144.6, 261.9; $\mathrm{P}$ $<0.001)$. Also, the agreement between straight-line and road network densities within $800(\mathrm{Kappa}=0.38)$ and $1000(\mathrm{Kappa}=0.47)$ metres were fair and moderate, respectively. The agreement between both methods to measure the proximity was fair to moderate $($ Kappa $=0.40)$. In addition, the correlation results showed that both the straight-line and road network proximity were negatively correlated to the accessibility score measured. Our findings suggest that the 800 and 1000 metres road network density and proximity may be more appropriate to explore the real relationships between fast food accessibility and diet or health relationships. In addition, the Hansen index is another metric that may be used if the aim of the study is to consider multiple locations when calculating the accessibility score. The availability of best-practice methods would help to explore the food environment in a consistent way and therefore lead to the implementation of effective actions and policies targeting the food environments, particularly around secondary schools.
\end{abstract}

\section{Conflict of Interest}

"There is no conflict of interest" 